\title{
Nuclear Transitions Induced by Synchrotron X-rays
}

\author{
Donald S. Gemmell \\ Physics Division, Argonne National Laboratory, Argonne, Illinois 60439 USA
}

\begin{abstract}
We discuss two rare but interesting processes by which synchrotron x-rays with energies up to about $100 \mathrm{keV}$ may be used to induce nuclear transitions.

In the NEET (Nuclear Excitation by Electronic Transition) process, an intense x-ray beam is employed to make vacancies, e.g. K-holes, in the atoms of a specific nuclear isotope. When a vacancy is filled by an electronic transition from a higher atomic level, there is some probability that instead of the usual x-ray or Auger emission, the nucleus of the atom itself will be excited. This is then followed by a nuclear decay exhibiting characteristic gamma-rays or other types of radiation, with time delays typical of the nuclear states involved. The probability for NEET increases when the energies of the atomic and the nuclear transitions become close. We address some theoretical aspects of the process and describe experimental efforts to observe it in ${ }^{189} \mathrm{Os}$ and ${ }^{197} \mathrm{Au}$.

The second process to be discussed is the possibility of "triggering" the decay of a nuclear isomer by irradiation with an x-ray beam. We focus on the case of the 31 -year, $2.4-\mathrm{MeV}, 16+$ isomer of ${ }^{178} \mathrm{Hf}$. There has been speculation that if one could isolate gram quantities, say, of this isomer and then have the capability to accelerate its decay in a controlled way, one would have a powerful triggerable source of enormous energy. This could be used to generate explosions, for rapid irradiations, or for more general energy-storage applications, depending on the rate of energy release. We describe attempts to observe this process.
\end{abstract}

\section{INTRODUCTION}

Transitions between energy levels of atomic nuclei can be induced by photons in various well understood ways. Examples include resonant absorption, Coulomb excitation, and inelastic electron scattering. (The latter two are mediated by virtual photons.) Here we discuss two processes, which occur with low probability, but which nevertheless are of interest from a fundamental point of view and may have significant potential for various applications. These are 1) NEET (Nuclear Excitation by Electronic Transition), which has only recently, after many erroneous claims by several groups over a period of almost thirty years, been definitively observed in the nucleus ${ }^{197} \mathrm{Au}[1]$, and 2) the acceleration of the decay of long-lived nuclear isomers by "triggering" with x-ray irradiation, a process that has been claimed [2 - 4] to have been observed in the second isomeric state $\left(16^{+}\right)$of the nucleus ${ }^{178} \mathrm{Hf}$, but that is disputed by other authors $[5,6]$. 
In this discussion we give particular emphasis to measurements made at the Advanced Photon Source (APS) at Argonne National Laboratory and at SPring-8 in Japan. At the APS we have looked for NEET in ${ }^{189}$ Os $[7,8]$ and have attempted to verify the $\mathrm{x}$-ray triggering of the decay of ${ }^{178 \mathrm{~m} 2} \mathrm{Hf}$ [6]. At SPring-8, Kishimoto et al.[1] have observed NEET in ${ }^{197} \mathrm{Au}$, and other experimenters [4] have reported the observation of triggering in ${ }^{178} \mathrm{~m} 2 \mathrm{Hf}$. Interestingly, this most recent claim to observe triggering in ${ }^{178 \mathrm{~m} 2} \mathrm{Hf}$ postulates that the process occurs via NEET.

\section{NEET}

The concept of the NEET process was introduced in 1973 by Morita [9], who pointed out that an atomic inner-shell vacancy might have an observable probability to decay by other than the "normal" modes of x-ray or Auger emission if the decay energy were to match closely an excitation to a state of that atom's nucleus. The associated changes in spin and parity of the atomic and nuclear transitions would also have to match. The energy of the atomic state would be transferred via the exchange of a virtual photon into excitation of the nucleus of that same atom, a process akin to the inverse of internal conversion. The NEET probability, $P_{N E E T}$, is defined as the probability that the decay of the initial excited atomic state will result in the excitation of (and subsequent decay from) the corresponding nuclear state. $P_{\text {NEET }}$, although generally small, is expected to be larger in heavier atoms (more compact atomic wave functions), such as the ${ }^{189} \mathrm{Os}$ and ${ }^{197} \mathrm{Au}$ considered here. Reference 10 gives a list of heavy atoms where the energy, spin, and parity requirements for atomic and nuclear transitions are simultaneously met and where, therefore, one might possibly detect NEET.

To calculate the magnitude of $P_{\text {NEET }}$, let us consider a heavy atom in which an electronic transition can occur between an initially excited $K$-vacancy state and a final $M$-vacancy state (a similar discussion applies to the case of an initial $L$-vacancy). This situation is illustrated in Fig. 1.

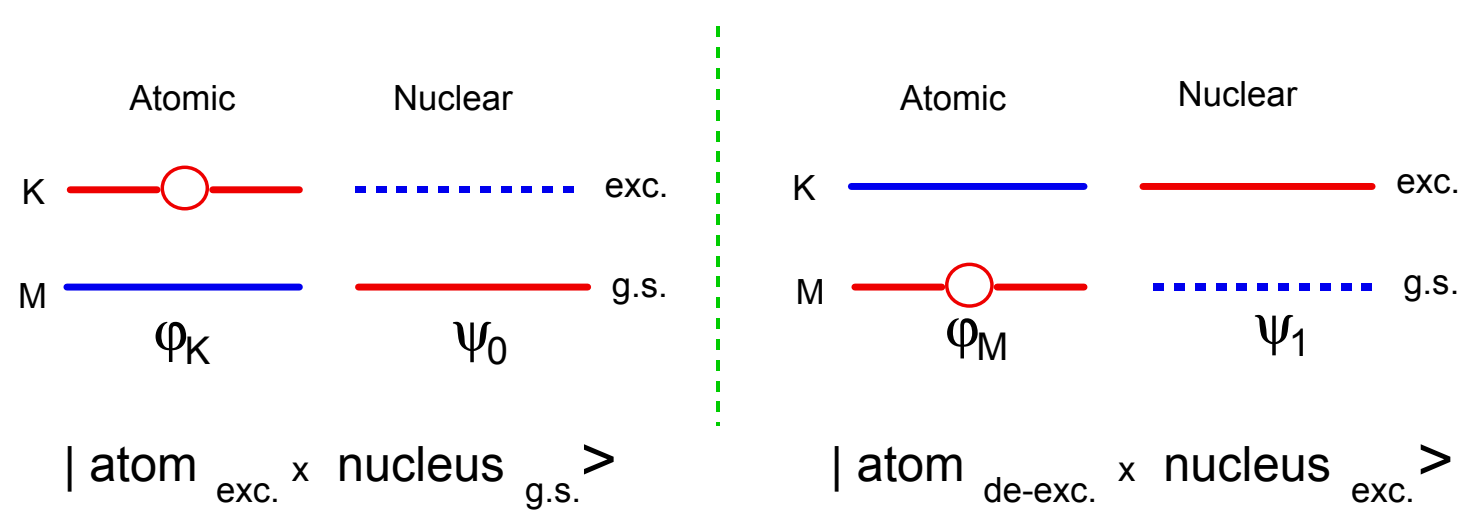

FIGURE 1. Initial and final states involved in a NEET transition. 
Let us assume that the nucleus of that atom can undergo an excitation to a level involving the same changes in angular momentum and parity as are involved in the atomic transition. NEET can occur when the two product states are nearly degenerate. They are coupled by a residual interaction, $V_{e m}$, the electromagnetic interaction of the electron hole with the protons in the nucleus. Let $\varphi_{i}$ denote the atomic wavefunction and $\psi_{j}$ the nuclear wavefunction. Following the creation of a $K$-hole, the initial state has a product wavefunction $|\alpha\rangle=\left|\varphi_{K} \psi_{0}\right\rangle$. The residual interaction generates an amplitude for the state $\left.|\beta>=| \varphi_{M} \psi_{l}\right\rangle$ and one can detect this component by measuring the nuclear decay. We can write the time evolution of the total wavefunction as

$$
|\Phi(t)\rangle=a_{\alpha}(t)|\alpha\rangle+a_{\beta}(t)|\beta\rangle \quad,
$$

where the amplitudes $a_{\alpha}$ and $a_{\beta}$ have initial $(t=0)$ values of 1 and 0 , respectively. We determine the two time-dependent amplitudes from the following coupled equations, which include the off-diagonal matrix element, $\boldsymbol{\kappa}=\left\langle\alpha\left|V_{e m}\right| \beta\right\rangle$, and the decay rates of both states explicitly:

$$
\begin{aligned}
& i \hbar \frac{d a_{\alpha}}{d t}=\left(E_{\alpha}-i \Gamma_{\alpha} / 2\right) a_{\alpha}+\kappa a_{\beta}, \\
& i \hbar \frac{d a_{\beta}}{d t}=\kappa a_{\alpha}+\left(E_{\beta}-i \Gamma_{\beta} / 2\right) a_{\beta},
\end{aligned}
$$

where $\left(E_{\alpha}, \Gamma_{\alpha}\right)$ and $\left(E_{\beta}, \Gamma_{\beta}\right)$ are the energies and decay widths of the two product states, $\mid \alpha>$ and $\mid \beta>$, respectively. The associated decay probabilities are

$$
P_{\alpha}=\frac{\Gamma_{\alpha}}{\hbar} \int_{0}^{\infty}\left|a_{\alpha}(t)\right|^{2} d t, \quad P_{\beta}=\frac{\Gamma_{\beta}}{\hbar} \int_{0}^{\infty}\left|a_{\beta}(t)\right|^{2} d t .
$$

The state $\mid \beta>$ can decay either by a nuclear decay or by an electronic transition from the $M$-vacancy. An electronic transition will, however, still result in a nuclear decay at a later time. Thus $P_{\beta}$ is equal to the "NEET probability."

The coupled equations (Eq. 2) for the coefficients, $a_{\alpha}$ and $a_{\beta}$, can be solved analytically [7] and this leads to an exact, if somewhat complex, expression for $P_{N E E T}$ $\left(=P_{\beta}\right)$. For small $\kappa$, this expression reduces to

$$
P_{N E E T}^{\kappa \rightarrow 0}=\frac{\Gamma_{\alpha}+\Gamma_{\beta}}{\Gamma_{\alpha}} \frac{\boldsymbol{\kappa}^{2}}{\left(E_{\alpha}-E_{\beta}\right)^{2}+\left(\frac{\Gamma_{\alpha}+\Gamma_{\beta}}{2}\right)^{2}} .
$$

Estimates of $P_{N E E T}$ for various atomic/nuclear systems have been given by several authors [9 - 19], beginning with Morita [9]. Many of the early estimates involved the use of simplifying approximations that led to results at considerable variance with Eq. 4. Also, it was not recognized at first that $P_{N E E T}$ tends to be significantly higher for $M I$ transitions than for $E 2$ transitions, mainly because of the involvement of atomic sstates, which leads to a stronger coupling. The more recent calculations of Tkalya [14, 16] give values that are close to those obtained from Eq. 4. 
Experiments designed to demonstrate NEET face three major problems:

(1) The values of $P_{N E E T}$ tend to be small (typically less than $10^{-7}$ ) and therefore one must generate a large number of initial vacancy states. This usually requires the use of very intense beams and highly sensitive detection techniques.

(2) Evidence needs to be established that the NEET-excited nuclear state has, in fact, been populated. This can be done by observing characteristic decay radiation from that state (as in the case of ${ }^{197} \mathrm{Au}$ ) or from a lower nuclear state to which the NEETexcited nuclear state decays (as in the case of ${ }^{189} \mathrm{Os}$ ). In both cases the decay times of the nuclear states involved can be employed as an additional identification of the nuclear transitions. (Observation of "prompt" radiation is generally infeasible because of difficulties with background radiation.)

(3) One has to be certain that the observed radiation is that which follows NEET transitions and not some other process(es). For example, using particle bombardment to generate the initial atomic vacancy state may also result in the Coulomb or inelasticscattering excitation of the same state - often much more intensely than that due to NEET. Also, higher-lying nuclear states may be excited, which decay through the "NEET state", mimicking the NEET process. The use of "white" synchrotron or bremsstrahlung photon beams can similarly result in the population of the nuclear "NEET state" or of higher states by resonant nuclear absorption, thereby producing misleading results.

Early attempts to demonstrate NEET suffered from one or more of these problems. The solution $[1,7,8,20]$ was to perform the experiments using an intense monochromatic $x$-ray beam with an energy just above the ionization energy needed to create the initial atomic vacancies, but with the energy carefully chosen not to coincide with excited nuclear states (due attention also being given to the energies of harmonics of the fundamental beam energy).

Much of the experimental and theoretical work has been dedicated to studies of the third excited state $\left(5 / 2^{-}\right)$of ${ }^{189} \mathrm{Os}$, lying at $69.537 \mathrm{keV}$ [21]. This state decays rapidly $(1.6 \mathrm{~ns})$ with a partial branch $\left(\sim 1.2 \times 10^{-3}\right)$ to a lower-lying metastable (6-hr. half-life) $9 / 2^{-}$state at $30.814 \mathrm{keV}$, which in turn decays primarily by internal conversion and can readily be measured. In the NEET process involving the 69.537$\mathrm{keV}$ state in ${ }^{189} \mathrm{Os}$ an initial atomic $K$-vacancy decays via an electronic transition from the $M$-shell. The $K M_{I}(70.822 \mathrm{keV}, M 1), K M_{I V}(71.840 \mathrm{keV}, E 2)$, and $K M_{V}(71.911$ $\mathrm{keV}, E 2)$ atomic transitions can contribute. The corresponding nuclear state at 69.537 $\mathrm{keV}$ can be excited via $M 1$ or $E 2$ transitions from the $3 / 2^{-}$nuclear ground state.

Following the derivation of Ref. [7], the theoretical expression for the electromagnetic ( $M 1$ or E2) coupling matrix element is

$$
\kappa^{2}=4 \pi e^{2} B\left(\Pi L, \frac{3}{2}^{-} \rightarrow \frac{5^{-}}{2}\right)\left\langle j_{K} \frac{1}{2} L 0 \mid j_{M} \frac{1}{2}\right\rangle^{2}\left(\frac{q^{L+1}\left|m_{\Pi L}(q)\right|}{(2 L+1) ! !}\right)^{2},
$$

where $\Pi L$ represents $M 1$ or $E 2$, and $q\left(=35.24 \AA^{-1}\right)$ is the wave number of the nuclear transition. The atomic matrix elements $m_{\Pi_{L}}(q)$, defined in Eq. 12 of Ref. [14], were calculated using wavefunctions from the "GRASP2" code [22], and tabulated values [21] of $\mathrm{B}(M 1)$ and $\mathrm{B}(E 2)$ were used for the nuclear transition. The Clebsch-Gordan 
coefficient refers to the total angular momenta $j_{K}$ of the K-vacancy and $j_{M}$ of the $M$-vacancy states.

Inserting the calculated values of $\kappa$ together with the atomic transition energies given above, and the calculated atomic level widths of Ref. [23], we obtain the following predictions for the NEET probabilities:

$$
P_{\text {NEET }}(M 1)=1.3 \times 10^{-10} \text {, and } P_{\text {NEET }}(E 2)=3.8 \times 10^{-13} \text {. }
$$

Our experiments at ANL on ${ }^{189}$ Os were performed using $\mathrm{x}$-ray beams from a wiggler operated by the Basic Energy Sciences Synchrotron Radiation Center [24] at the APS. After initial measurements with a white beam, in which the difficulties mentioned above became very apparent, we switched to the use of a monochromatic 98.74-keV x-ray beam to produce the $K$-vacancy states. This beam $\left(5 \times 10^{11}\right.$ photons/s) was formed by Bragg diffraction from a single (440) Si crystal placed in the wiggler beam. The diffraction angle was $2 \theta=7.5^{\circ}$. This particular beam energy was chosen because it lies above the osmium $K$-edge at $73.9 \mathrm{keV}$, does not lie near the energy of a nuclear level in ${ }^{189} \mathrm{Os}$, and corresponds to a convenient and intense diffraction. The energy width of the beam was about $0.1 \%(100 \mathrm{eV})$ and the beamspot size at the target was $0.2-\mathrm{mm}$ wide and 4-mm high. The incident $\mathrm{x}$-ray beam contained a comparably intense component at $49.37 \mathrm{keV}$ (below the osmium K-edge) and also a few-percent component at $148.1 \mathrm{keV}$. None of the beam components had energies overlapping any of the ${ }^{189}$ Os nuclear level energies, thereby avoiding problems with nuclear resonant absorption.

The decay of the 30.814-keV metastable nuclear state was measured off-line using a Ge (LEPS) detector to count the $L$ x-ray spectrum associated with the $L$-conversion electrons. Figure 2(a) shows the $L$ x-ray spectrum obtained in the initial runs using a white beam. The inset shows a corresponding measurement of the decay of this radiation. The half-life of the $30.8-\mathrm{keV}$ state was measured to be $5.65 \pm 0.15 \mathrm{~h}$, in good agreement with the tabulated value [21] of $5.8 \pm 0.1 \mathrm{~h}$.

The monochromated x-ray beam from the wiggler was incident upon a thin $(9.3$ $\mathrm{mg} / \mathrm{cm}^{2}$ ) layer of isotopically separated $(95.3 \%)$ metallic ${ }^{189}$ Os electroplated onto a 0.015"-thick $\mathrm{Cu}$ disk using the method of Stuchbery [25]. Individual targets were irradiated in this fashion for periods of about 20 hours. Large numbers of $K$-vacancies were produced, some of which were expected to lead via NEET to the $69.5-\mathrm{keV}$ state and thence to the $30.8-\mathrm{keV}$ metastable state of the nucleus. The number of Os $K$-vacancies generated was monitored by on-line observation of the $K$ x-rays using a Ge detector.

After irradiation the targets were removed and the $L$ x-rays associated with decays of the metastable state were detected in a low-background shielded underground counting room where counting with a Ge detector proceeded also for about 20 hours. Figure 2(b) shows the results summed for two targets. From a comparison with Fig.2(a), it is apparent that within the sensitivity of this measurement, there was no evidence of the X-rays that accompany the decay of ${ }^{189}$ Os metastable state (the peak at 
$10.3 \mathrm{keV}$ in Fig. 2(b) is due to very weak natural background radiation and is only observable in extremely well shielded conditions).

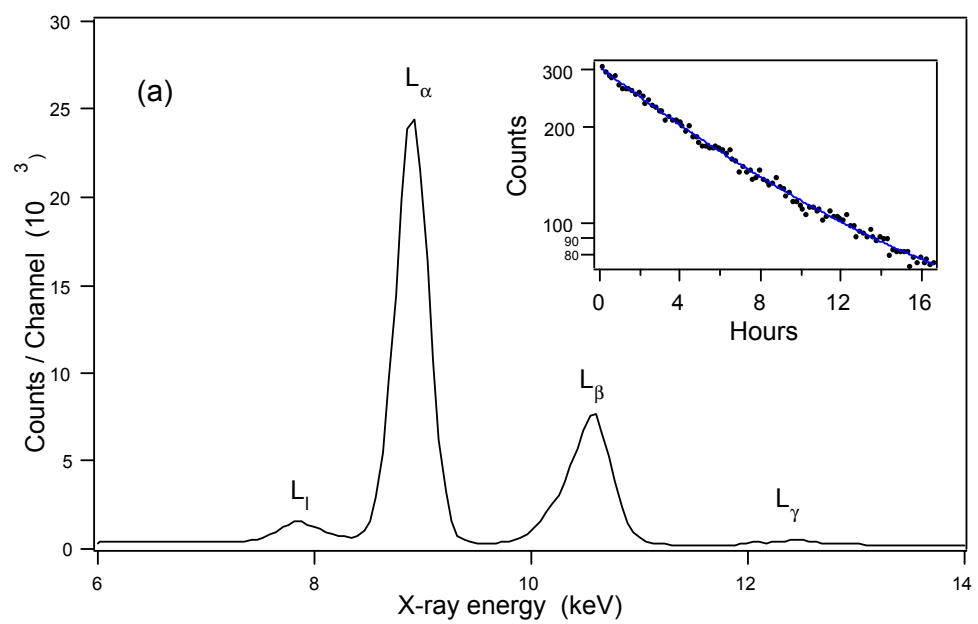

FIGURE 2. a) The osmium L X-ray spectrum obtained in the initial runs after irradiation with a white $\mathrm{x}$ ray beam. (In this case, the excitation of the $30.8-\mathrm{keV}$ state followed resonant nuclear absorption - - not NEET.) The inset shows a corresponding measurement of the 6-hr. decay of this radiation. b) The $\mathrm{x}$-ray spectrum summed for two targets irradiated with monochromatic $98.74-\mathrm{keV}$ $\mathrm{x}$-rays as described in the text. The total irradiation time was 43.9 hours and the total counting time was 37.4 hours.

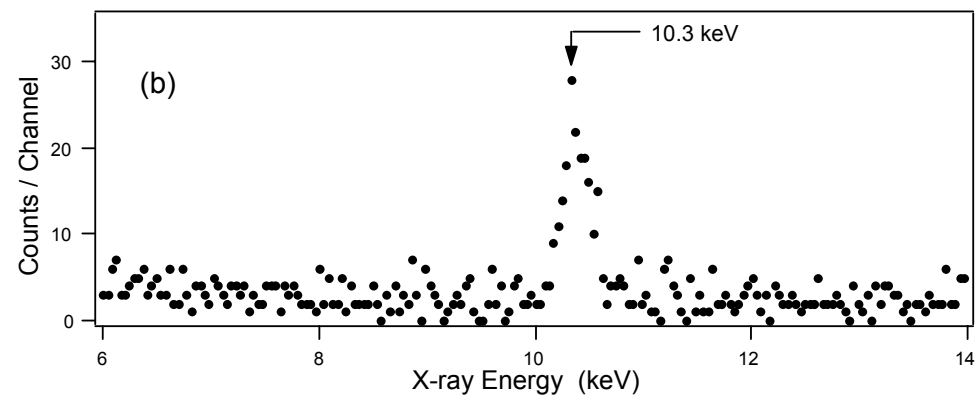

After taking into account factors such as the number of $K$-holes created during the irradiation (and their decay), the branching ratio for feeding the metastable state from the $69.5-\mathrm{keV}$ state, geometrical factors, the emission probability for $L$ x-rays in the isomeric decay, self-absorption in the target, etc, we obtained the result [7] $P_{N E E T}<9 \times 10^{-10}$. In a subsequent measurement with multiple targets and improved detection sensitivity [8], we were able to reduce this upper limit even further to $P_{N E E T}<3 \times 10^{-10}$. In more recent work at SPring-8, Aoki et al.[20], using a similar technique found $P_{N E E T}<4.1 \times 10^{-10}$. All of these values are significantly smaller than the various values obtained in previous measurements and predicted by previous calculations (see a summary in Ref. [7]). They are, however, consistent with our calculated value (Eq. 6) of $P_{\text {NEET }}(M 1)=1.3 \times 10^{-10}$ and also the recent values calculated by Tkalya $[14,16]\left(P_{N E E T}=1.2 \times 10^{-10}\right)$ and by Harston [19] $\left(P_{N E E T}=1.1\right.$ $\mathrm{x} 10^{-10}$ ).

The small value of $P_{\text {NEET }}$ in ${ }^{189}$ Os $\left(\sim 10^{-10}\right)$, which has made the process undetectable so far, is mainly due to the poor energy matching between the nuclear and atomic transitions. The mismatch is about $1.3 \mathrm{keV}$, or about $2 \%$ of the transition energy. A much more favorable case is found in ${ }^{197} \mathrm{Au}$, where the energy of the transition from the $3 / 2^{+}$nuclear ground state to the $77.351-\mathrm{keV} 1 / 2^{+}$first excited state 
differs by only $51 \mathrm{eV}(0.07 \%)$ from that of the $K \rightarrow M_{1}$ atomic hole transition. Both transitions are $M 1 / E 2$. However, the excited nuclear state has a half-life of only $1.91 \mathrm{~ns}$ [26] and so the decay radiation from the state (mainly $L$ internal-conversion electrons) is extremely difficult to measure in a situation where the prompt background radiation from a synchrotron is overwhelming.

In an outstanding technical tour-de-force, Kishimoto et al.[1] have succeeded in measuring $P_{N E E T}$ in ${ }^{197} \mathrm{Au}$. At SPring-8 this group exploited the pulsed nature of a monochromatic $(80.989-\mathrm{keV}) \mathrm{x}$-ray beam from an undulator. The pulses were of $50 \mathrm{ps}$ duration, spaced 42 ns apart. Using a silicon avalanche diode to detect the internalconversion electrons from excited nuclei, the group was able to measure time spectra showing the characteristic decay time $(2.8 \mathrm{~ns})$ of the $77.351-\mathrm{keV}$ nuclear state. The largest source of background was the very low-level $\left(\sim 10^{-6}\right.$ of the main bunch intensity) occupation of beam "buckets", spaced about 2 ns apart during the 42-ns periods between the main bunches. The NEET probability was found to be $P_{N E E T}=$ $(5.0 \pm 0.6) \times 10^{-8}$, in good agreement with the recently calculated values of Tkalya [27] $\left(3.8 \times 10^{-8}\right)$ and Harston [19] $\left(3.6 \times 10^{-8}\right)$, and in fair agreement with the results of Ljubicic [28] $\left(7.2 \times 10^{-9}\right)$, and Sumi and Tanaka [18] $\left(1.1 \times 10^{-7}\right)$.

The work of Kishimoto et al.[1] stands as the only definitive and unequivocal experimental demonstration to date of the phenomenon of NEET.

\section{"TRIGGERING" IN ${ }^{178 ~ m 2}$ HF}

The possibility of storing energy in nuclear isomers has been discussed for many years. One of the most favorable candidates is considered to be the 2.4-MeV, 16+, 31year level in ${ }^{178} \mathrm{Hf}$. This isomeric state, designated ${ }^{178 \mathrm{~m} 2} \mathrm{Hf}$, is produced among the residual activities in nuclear spallation reactions on target materials like tantalum. The attractive notion is that if one could isolate gram quantities, say, of this isomer and then have the capability to trigger its decay so that the nuclei all dump their $2.4-\mathrm{MeV}$ of gamma-ray energy in a fraction of a second rather than gradually over 31 years, one would have a powerful triggerable source of very high energy content (one gram of the isomer would contain a releasable energy of 1.3 Gigajoules, equivalent to about one third of a ton of TNT). This could be used for explosions, for rapid irradiations, or for more general energy-storage applications, depending on the rate of energy release.

This topic remained one of primarily academic interest until 1999 when a group at the University of Texas at Dallas published [2] the results of experiments in which they observed about a $4 \%$ increase in the decay rate of the ${ }^{178 \mathrm{~m} 2} \mathrm{Hf}$ isomer when it was irradiated by a broad spectrum of x-rays from a dental x-ray machine. Over the next couple of years the same group published further results [3] basically reinforcing those in their original publication. These results generated a great deal of discussion (see, for example, Ref. [29]) and there was speculation on applications in gamma-ray lasers, countering bio-terror attacks, energy storage, etc. 
In its normal decay mode, the $16^{+}$isomeric state decays primarily through an E3 transition to the $13^{-}$member of the $K=8$ band whose $J^{\pi}=8^{-}$bandhead is itself an isomer (half-life $=4 \mathrm{~s}$.), decaying through a cascade in the ground-state band (Fig. 3 ).

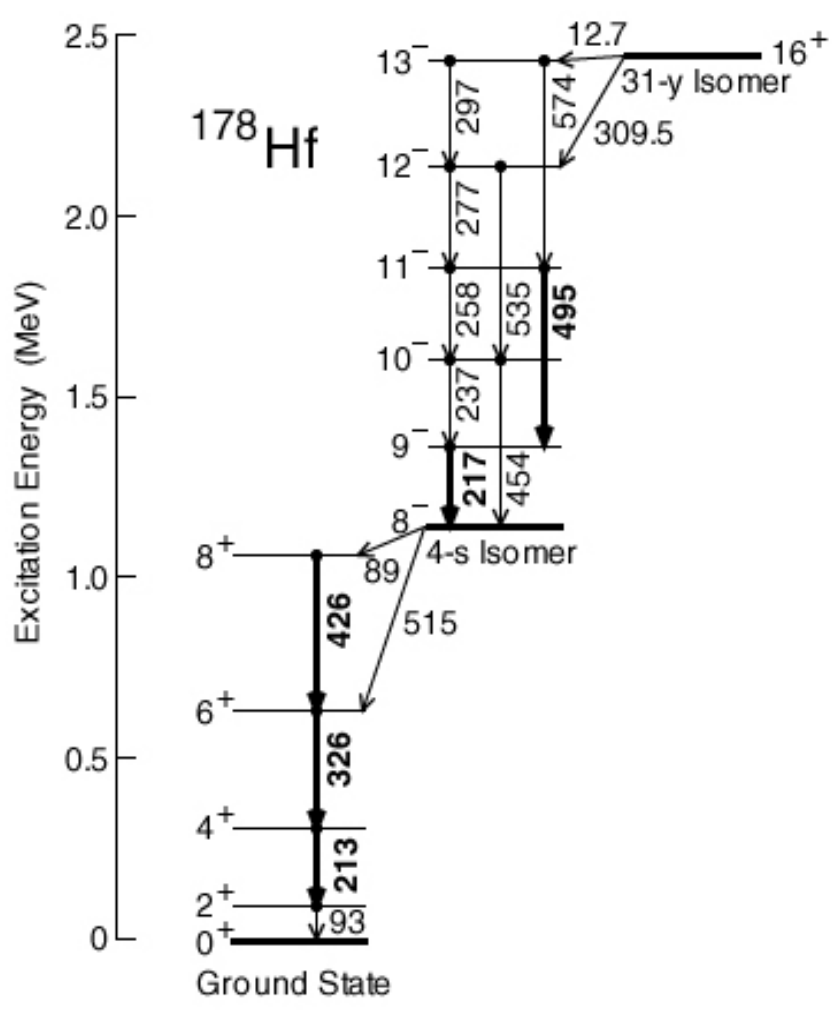

FIGURE 3. Energy level diagram showing the decay of the $31-\mathrm{yr}{ }^{178} \mathrm{Hf}$ isomer. The transition energies are labelled in $\mathrm{keV}$. Those transitions reported in [2-4] to be enhanced are highlighted.

The mechanism proposed by Collins et al.was that $\mathrm{x}$-rays are resonantly absorbed by ${ }^{178} \mathrm{~m} 2 \mathrm{Hf}$ into a state (or states) of mixed $K$ lying about 20 to $60 \mathrm{keV}$ above the isomeric state. Such a state could then decay promptly to a lower-lying level in the $K=8$ band and thence rapidly to the $J^{\pi}=8^{-}$bandhead, which in turn decays in a well understood cascade to the nuclear ground state. A drawback with this explanation is that the measurement implies that the initial resonant absorption has an integrated cross section $\left(1 \times 10^{-21}\right.$ $\left.\mathrm{cm}^{2} \mathrm{keV}\right)$, which exceeds the values normally found for nuclear photoabsorption in this mass region by about 7 orders of magnitude [5].

An attempt to confirm this acceleration of the decay rate in $178 \mathrm{~m} 2 \mathrm{Hf}$ was made last year by a collaboration of scientists from Argonne, Los Alamos, and Livermore national laboratories [6]. This group used the APS light source to provide beams of x-rays with intensities that were over 4 orders of magnitude greater than those provided by the dental x-ray machine of Collins et al. A "white" beam from a tapered undulator at the SRI-CAT 1-1D beam line was used. Tapering the undulator gap and using two different average gap settings $(15 \mathrm{~mm}$ and $20 \mathrm{~mm}$ ) allowed us to smooth out the otherwise sharp energy structure inherent in undulator radiation and to be sure that there were no "holes" in our beam energy coverage in the range from about $8 \mathrm{keV}$ to over $100 \mathrm{keV}$. The size of the beam spot at the target, $37 \mathrm{~m}$ from the undulator, was $2 \times 2 \mathrm{~mm}$. During the measurement, the stored electron beam at the APS was maintained at a steady $100 \mathrm{~mA}$ through the use of a continuous "top-up" mode of operation.

We employed three separate targets containing $7.3 \times 10^{14}, 3.0 \times 10^{15}$, and $6.4 \mathrm{x}$ $10^{15}$ isomeric ${ }^{178} \mathrm{Hf}$ nuclei, respectively. The Hf was produced at LANL using the LANSCE accelerator to induce $800-\mathrm{MeV}$ proton spallation reactions on thick Ta targets/beam stops. The Hf was chemically extracted from the Ta target material, 
purified, precipitated and fired to produce $\mathrm{HfO}_{2}$ for use in the current experiment. In addition to the 31-year ${ }^{178} \mathrm{Hf}$ isomer, the target contained some stable $\mathrm{Hf}$ from impurities in the beam stop. The spallation reaction also produced other Hf isotopes, including trace amounts of ${ }^{172} \mathrm{Hf}\left(t_{1 / 2}=1.89 \mathrm{yr}\right)$, that proved useful in monitoring the target during the experiment. For each of the three targets, the hafnium oxide was mixed with fine aluminum powder (for heat conduction purposes - - the beam typically deposited $\sim 200 \mathrm{~W}$ into the $\mathrm{HfO}_{2}$ ) and then sealed by electron-beam welding into a 2$\mathrm{mm}$ diameter by $1.6-\mathrm{mm}$ deep cavity machined into a water-cooled aluminum block. The aluminum entrance window for each target was $0.15 \mathrm{~mm}$ thick.

$\mathrm{X}$-rays and gamma rays from the target were measured with two heavily shielded planar Ge detectors at $90^{\circ}$ to the beam. The Hf $K$ X-rays were used to monitor the target. Gamma-ray spectra were accumulated and time-sorted as the incident beam was cycled on (for $11 \mathrm{~s}$.) and off (for $22 \mathrm{~s}$., divided into two equal periods of $11 \mathrm{~s}$.). In

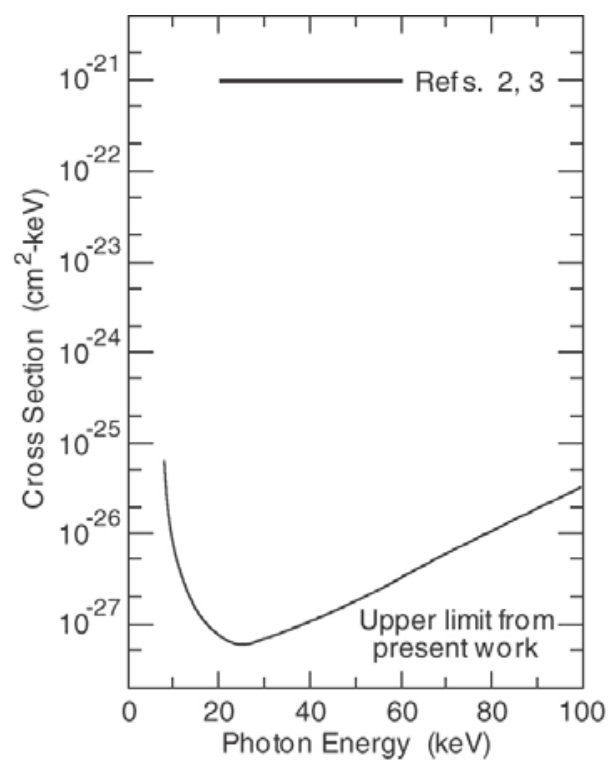

FIGURE 4. Upper limit of the integrated cross section for photoninduced de-excitation of the $31-\mathrm{yr}{ }^{178} \mathrm{Hf}$ isomer through the $4-\mathrm{s}, 8^{-}$isomer. The corresponding value reported by Collins et al. $[2,3]$ is also shown. this way we were able to search for both prompt and delayed (through the $J^{\pi}=8^{-}$ bandhead) enhancements of the ${ }^{178 \mathrm{~m} 2} \mathrm{Hf}$ decay rate. (A beam-induced decay of the isomer would result in an increased production of the 4-s. isomer and the emission of the subsequent gamma rays in the ground-state band would be enhanced in the first half of the beam-off period as compared to the second.)

Based on the results of Refs [2, 3] (a $\sim 4 \%$ enhancement of the decay rate due to resonant photon absorption in the range $20-60 \mathrm{keV}$ ), the decay rates observed in our experiment should have been enhanced by a factor of $\sim 20,000$. Instead, we saw that the decay rate was constant to within our uncertainty of $\pm 2 \%$, i.e. about a million-fold smaller than would be consistent with Refs [2,3]. Our result implies an integrated photon absorption cross section of less than $2 \times 10^{-27} \mathrm{~cm}^{2} \mathrm{keV}$ in the $20-60$ $\mathrm{keV}$ range, a value much more in keeping with the accumulated body of knowledge of gamma-ray transition strengths in this mass and energy region. Figure 4 shows our upper limit as a function of photon energy.

At low energies, the upper limit rises steeply due to (a) the rapid rise of absorption in the target material, and (b) the fact that the incident beam loses flux at energies below about $8 \mathrm{keV}$ (due to absorption effects in various windows in the beam line).

Although our measurement was designed primarily to address the question of resonant absorption in the originally reported $20-60 \mathrm{keV}$ range, our data, as indicated in Fig. 4, cover a somewhat wider energy region. At the extreme low-energy portion of this range $(\sim 8 \mathrm{keV})$, the difficulties mentioned above come into play. For this 
reason, and to obtain more accurate data at energies around $5-20 \mathrm{keV}$, we have recently performed additional measurements [30] using thin targets of pure $\mathrm{HfO}_{2}$ (containing ${ }^{178 \mathrm{~m} 2} \mathrm{Hf}$, as before) sandwiched between thin Be foils. These $\mathrm{HfO}_{2}$ targets were thin compared to the mean-free-path of $8-\mathrm{keV}$ photons. Again, no enhancements were observed. A preliminary analysis shows that the upper limit for the integrated cross section in the range $7-30 \mathrm{keV}$ is everywhere less than $8 \times 10^{-27} \mathrm{~cm}^{2} \mathrm{keV}$. At $8 \mathrm{keV}$, the upper limit has been reduced to $5 \times 10^{-28} \mathrm{~cm}^{2} \mathrm{keV}$, a factor of 100 lower than obtained in our previous measurement (Fig. 4).

In a recent publication [4], Collins et al.show results obtained by scanning a monochromatic x-ray beam at SPring- 8 over the energy range $9-13 \mathrm{keV}$. Here too, they claim to observe x-ray induced acceleration of the decay of ${ }^{178} \mathrm{~m} 2 \mathrm{Hf}$. The enhancements range from $1 \%$ to $3 \%$. In this case, the authors postulate that the mechanism is NEET, involving initial $L$-vacancies created by the incident photons. The enhancements appear to track the $L$-absorption yields. Just above the $L_{1}$ edge, for example, their measurements imply $P_{\text {NEET }}=2 \times 10^{-3}$, which vastly exceeds any other measured or calculated value. For instance, Tkalya $[16,31]$ calculates that even in the extremely favorable (and unlikely) case in which the atomic and the nuclear energies match exactly, and assuming that the $(E 1)$ NEET transition proceeds through the $K$ mixed state with a reduced transition probability of 0.01 Weisskopf units (the upper limit for transitions in this mass region), then one expects a value $P_{N E E T} \approx 10^{-20}$. This includes a factor of $\sim 10^{-14}$ to take into account the admixture of $\Delta K=8$ in the intermediate state.

In summary, it appears that attractive as the possibility of "triggered" decay of isomers may seem, the accuracy of the measurements of Collins et al.[2 - 4] do not provide convincing evidence that the process has been observed. The measurements made at the APS indicate upper limits in the case of ${ }^{178 ~}{ }^{2} \mathrm{Hf}$ that are consistent with known nuclear parameters in this region of mass and energy.

\section{ACKNOWLEDGEMENTS}

This work was supported in part by the U.S. Department of Energy (DoE) under contract No. W-31-109-ENG-38 (ANL), in part by the DoE under contract Nos. W7405-ENG-48 (UC-LLNL), and W-7405-ENG-36 (LANL), and in part by the DoE Nuclear Energy Research Initiative (NERI). We are indebted to J. Greene, T.L. Khoo, C.J. Lister, R. Nelson, W. Patterson, and K. Teh for their help in various aspects of these measurements, to D.R. Haeffner, D.M. Mills, and G. Shenoy for their encouragement of this investigation at the SRI-CAT 1-ID beamline, and to E. V. Tkalya for his theoretical assistance. 


\section{REFERENCES}

1. Kishimoto, S., Yoda, Y., Seto, M., Kobayashi, Y., Kitao, S., Haruki, R., Kawauchi, T., Fukutani, K., and Okano, T., Phys. Rev. Lett. 85, 1831 (2000).

2. Collins, C.B., Davanloo, F., Iosif, M.C., Dussart, R., Hicks, J.M., Karamian, S.A., Ur, C.A., Popescu, I.I., Kirischuk, V.I., Carroll, J.J., Roberts, H.E., McDaniel, P., and Crist, C.E. Phys. Rev. Lett. 82, 695 (1999).

3. Collins, C.B. et al., Laser Phys. 9, 8 (1999); Collins, C.B. et al., Phys. Rev. C 61, 054305 (2000); Collins, C.B. et al., Phys. At. Nucl. 63, 2067 (2000).

4. Collins, C.B. et al., Europhys. Lett., 57, 677 (2002).

5. Olariu, S., and Olariu, A., Phys. Rev. Lett., 84, 2541 (2000); McNabb, D.P. et al., Phys Rev. Lett., 84, 2542 (2000); von Neumann-Cosell, P., and Richter, A., Phys. Rev. Lett., 84, 2543 (2000).

6. Ahmad, I., Banar, J.C., Becker, J.A., Gemmell, D.S., Kraemer, A., Mashayekhi, A., McNabb, D.P., Miller, G.G., Moore, E.F., Pangault, L.N., Rundberg, R.S., Schiffer, J.P., Shastri, S.D., Wang, T.F., and Wilhelmy, J.B., Phys. Rev. Lett., 87, 072503 (2001).

7. Ahmad, I., Dunford, R.W., Esbensen, H., Gemmell, D.S., Kanter, E.P., Rütt, U., and Southworth, S.H., Phys. Rev. C 61, 051304(R) (2000).

8. Ahmad, I., Dunford, R.W., Gemmell, D.S., Lister, C.J., Siemssen, R.H., and Southworth, S.H., (2000, unpublished).

9. Morita, M., Prog. Theor. Phys. 49, 1574 (1973)

10. Okamoto, K., Laser Interactions and Related Phenom. 4 A, 283 (1977).

11.Pisk, K., Kaliman, Z., and Logan, B.A., Nucl. Phys. A 504, 103 (1989).

12. Bondar'kov, M.D., and Kolomietz, V.M., Izv. Akad. Nauk. SSSR, Ser. Fiz. 55, 983 (1991).

13.Ljubicic, A., Kekez, D., and Logan, B.A., Phys. Lett. B 272, 1 (1991).

14. Tkalya, E.V., Nucl. Phys. A 539, 209 (1992).

15. Ho, Y-k., et al, Phys. Rev. C 48, 2277 (1993).

16. Tkalya, E.V., "Nuclear Excitation by Electronic Transition Between Atomic Shells," in X-Ray and Inner-Shell Processes, edited by R.W.Dunford et al., AIP Conference Proceedings 506, Melville, New York, 2000, pp.486-495.

17. Ljubicic, A., "Excitations of Nuclear Levels in Atomic Transitions," in Proceedings of the International Meeting on Frontiers of Physics 1998, Kuala Lumpur, 26 - 29 October 1998, edited by S.P. Chia and D.A. Bradley, World Scientific, pp. $133-141$.

18. Sumi, Y., and Tanaka, S., Jpn. J. Appl. Phys. 39, 1894 (2000).

19. Harston, M.R., Nucl. Phys. A 690, 447 (2001).

20. Aoki, K., et al., Phys. Rev. C 64, 044609 (2001).

21. Firestone, R.B., Nucl. Data Sheets, 59, 869 (1990).

22.Parpia, F.A., Fischer, C.F., and Grant, I.P., Comput. Phys. Commun. 94, 249 (1996).

23. See, for example, Leisi, H.J., et al, Helv. Phys. Acta, 34, 161 (1961), and McGuire, E.J., Phys. Rev. A 5, 1043 (1972).

24. Montano, P.A., et al, Rev. Sci. Instrum. 66, 1839 (1995).

25. Stuchbery, A.E., Nucl. Instrum. Methods. 211, 293 (1983).

26. Chunmei, Z., Nucl. Data Sheets, 62, 433 (1991).

27. Tkalya, E.V., Ref. [16], corrected for the omission of a small numerical factor (Private Communication).

28. Ljubicic, A., Ref. [17], corrected for a printing error (Private Communication).

29. SCIENCE, 283, 769 (1999).

30. Ahmad, I., Banar, J.C., Becker, J.A., Bredeweg, T., Cooper, J., Gemmell, D.S., Mashayekhi, A., McNabb, D.P., Moore, E.F., Palmer, P.D., Rundberg, R.S., Schiffer, J.P., Shastri, S.D., Wang, T.F., and Wilhelmy, J.B., (to be published).

31. Tkalya, E.V., (Private Communication). 